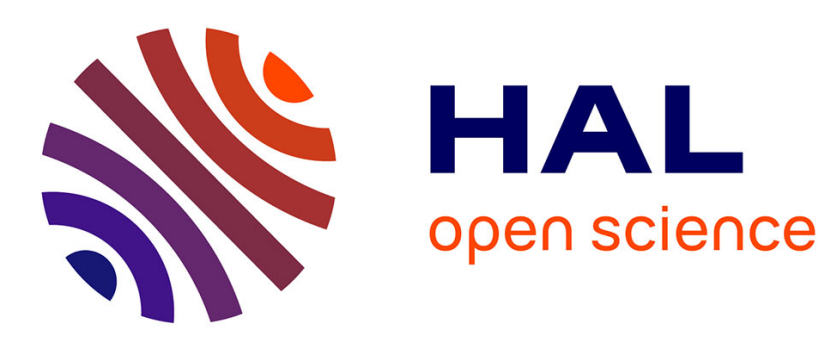

\title{
Optimal sparse apertures for phased-array imaging
}

Frederic Cassaing, Laurent Mugnier

\section{To cite this version:}

Frederic Cassaing, Laurent Mugnier. Optimal sparse apertures for phased-array imaging. Optics Letters, 2018, 43 (19), pp.4655-4658. 10.1364/OL.43.004655 . hal-02061419

\section{HAL Id: hal-02061419 https://hal.science/hal-02061419}

Submitted on 8 Mar 2019

HAL is a multi-disciplinary open access archive for the deposit and dissemination of scientific research documents, whether they are published or not. The documents may come from teaching and research institutions in France or abroad, or from public or private research centers.
L'archive ouverte pluridisciplinaire HAL, est destinée au dépôt et à la diffusion de documents scientifiques de niveau recherche, publiés ou non, émanant des établissements d'enseignement et de recherche français ou étrangers, des laboratoires publics ou privés. 


\title{
Optimal sparse apertures for phased-array imaging
}

\author{
F. Cassaing ${ }^{1, *}$ AND L. M. MUgnier ${ }^{1}$ \\ ${ }^{1}$ ONERA / DOTA, Université Paris Saclay, F-92322 Châtillon - France \\ *Corresponding author: Frederic.Cassaing@onera.fr
}

Compiled March 1, 2019

\begin{abstract}
A key issue in a sparse-aperture imaging system is the relative arrangement of apertures, or aperture configuration. Transposing previous works into a discrete setting, we perform a systematic search for maximalresolution configurations with 9 to 21 apertures. From the catalog of found solutions, we derive a procedure to simply optimize the main free parameters of the aperture from high-level constraints, such as the sought resolution and the minimum MTF level or the fill-factor.

(C) 2019 Optical Society of America
\end{abstract}

OCIS codes: (110.1220) Apertures; (110.5100) Phased-array imaging systems; (110.3175) Interferometric imaging; (110.4100) Modulation transfer function.

Pioneered by Fizeau [1], sparse-aperture imaging systems (SAISs) are now used for astronomy [2,3] and considered for spaceborne imaging $[4,5]$ or ground-based satellite imaging $[6,7]$. Based on pupil masks [2], segmented primary [4], telescope arrays [5-9], photonics chips [10] or laser illumination [11], SAISs can access very high resolution, reject atmospheric turbulence, or reduce the system size, weight and power (SWAP). But unlike for single-aperture telescopes, the array performance strongly depends on many free parameters to be optimized during the design: the number, position, and diameter of the sub-apertures, known as aperture configuration.

This Letter proposes a methodology to quickly but optimally define a SAIS from a small set of high-level requirements. Main parameters and properties of the SAIS are first recalled, performance criteria are then discussed, and a catalog of optimal aperture configurations found by an exhaustive search is presented and its use through simple formulas illustrated.

Three main types of arrays can be distinguished, in relation to the shape of their modulation transfer function (MTF) given by the aperture autocorrelation $(\otimes)$, up to a wavelength scaling factor omitted in the following. The first type is the segmented telescope, with nearly contiguous sub-apertures to maximize the collecting area for a given resolution [12]. The global aperture is then hardly distinguishable from a connex, nearly filled, aperture and the MTF very close to that of a classical telescope. A second type is the stellar interferometer, with metric sub-apertures spanning hectometers for very high resolution [13]. This results in a diluted pupil, in the sense that the instantaneous frequency coverage is partial, even if complemented by Earth rotation. The small number of observables has, until the last decade, often

limited its use to the estimation by model fitting of a few stellar parameters [14]. Additionally, the discrete frequency sampling by the MTF leads to field aliasing, restricting the interest of this observation mode to objects with small support. We will focus here on the intermediate third type of array (Fig. 1), where the aperture is made sparse to enable both complex images and maximal resolution with minimum SWAP, at the expense of exposure time [15]. Such SAISs include focal-plane imagers [4, 5, 7-9], where the pupil is necessarily compact, i.e., the MTF is non-null up to a cutoff frequency defined as the practical resolution limit (PRL) [16].

The design of a SAIS aperture configuration has been widely addressed in the radio and optical domains [16-19]. A first catalog is due to Golay [17], who identified a dozen widely used configurations discussed hereafter. Another catalog has been established with a priori configurations [20]. Some works perform a metric-based aperture configuration optimization, but the metric definition is often based on somewhat ad hoc criteria [21], and even if the chosen metric uses the MTF shape or support it does not consider explicitly the noise propagation occurring during the necessary image restoration [22]. For wide-field imaging, a rigorous approach is to consider that the optimal aperture minimizes the Euclidian distance $\epsilon$ between the observed object and the object restored from its image, cf. Eq. (4) of [18]. Simplifying assumptions leads to the main result that $\epsilon$ is proportional to the inverse of $\mathrm{MTF}_{\min }$, the MTF minimum over the frequency domain of interest (FDI), cf. Eq. (16) of [18]. This precisely defines the commonly acknowledged MTF uniformity requirement and demonstrates the compactness requirement, but does not give an explicit solution for the best aperture. Unfortunately, the large number of continuous free parameters makes an exhaustive search difficult for a large number of sub-apertures, and many suboptimal configurations exist since the criterion

a)

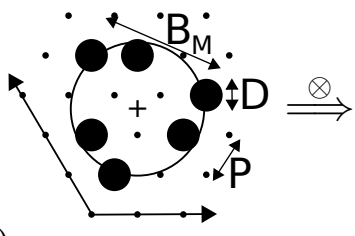

Fig. 1. Example of sparse aperture (a) and MTF (b, logarithmic scale) for a Golay-6 array of dilution 1.4 ; $P$ : grid pitch; $D$ : subaperture diameter; $B_{\mathrm{M}}$ : maximum baseline; +: PA center. 
defined by [18] is not convex. When using the tool developed in [18] with continuous positions, the optimum sub-aperture positions were found to be close to triangular lattices [23]. The core of this Letter is to transpose [18] to a lattice and to perform a systematic search, since quantifying sub-aperture positions on a lattice considerably reduces the number of degrees of freedom.

We will consider here arrays of $N$ identical sub-apertures of diameter $D$, and call point-array (PA) the set of their centers. We will restrict ourselves to PAs with integer coordinates on a regular lattice of pitch $P$ [Fig. 1(a)]. Such lattices can be made only of squares, regular hexagons or triangles. Since hexagonal lattices are a sub-set of triangular lattices, and square lattices lead to poor paving, we focus (as Golay did) on triangular lattices. Then, the free aperture parameters boil down to the normalized (unitary pitch) PA, the actual pitch $P$, the dilution ratio $d_{L}=$ $P / D[16]$ and marginally the sub-aperture shape (disk/hexagon, central obscuration, etc.). Assuming that there are no aberrations, or that they are later accounted for as a MTF reduction, the total aperture transmittance is the convolution of a single subaperture transmittance by the PA.

The resulting MTF is the convolution of the PA autocorrelation (PAA), by the inter-correlation between two sub-apertures, which is also the single-aperture MTF. It is made of a central peak (sum of the $N$ sub-aperture autocorrelations) whose height is normalized to 1 , surrounded by $(N-1) N$ fringe peaks of height $1 / N$ associated to each baseline formed by a sub-aperture pair [Fig. 1(b)]. The PAA is on the same lattice as the PA, but each fringe peak has a support of diameter $2 D$. Thus fringe peaks are disjoint when $d_{L} \geq 2$ and their overlapping can be continuous if $d_{L} \leq \sqrt{3}$ [as in Fig. 1(b)]. The PAA can be characterized by the distance $D_{\text {PRL }}$ from its center to the first zero, which we will approximate for simplicity by the distance from the origin to the closest lattice point outside the PAA [16].

A MTF like the one in Fig. 1(b) has strong system impacts. First, an image processing step must be included in the system to equalize the oscillating plateau and local peaks with respect to the central peak. Second, unlike full-aperture systems with a monotonically decreasing MTF, the Nyquist frequency is ideally placed near the PRL where the MTF has a steep cutting slope: a smaller value would introduce aliasing and wastage (baselines longer than required are built), and a larger value would require more pixels than useful, thus more noise and a smaller field. Third, for wide-field imaging, a continuous frequency coverage-thus, a sufficiently small dilution-is required. Incidentally, since adjacent MTF peaks must constructively superimpose for continuous frequency coverage, a co-phasing subsystem is required to phase the array apertures.

An equivalent dual approach to the maximization of the MTF over a given FDI in a continuous setting, as in [18] but in a discrete setting, is to maximize the PRL of a unit-pitch latticebased PA. Indeed, scaling the PA to the actual radius of the FDI, after this maximization, will yield a minimal pitch and thus a maximal value for $\mathrm{MTF}_{\text {min }}$.

An important result is that PAs that are both non-redundant, i.e., where all fringe peaks are formed at different frequencies, and compact, such as Golay-6 in Fig. 1, unfortunately no longer exist as soon as $N>6$. In his search for non-redundant PAs (Fig. 2), Golay identified the $a$ series where all peaks are inside a central core but with holes inside this core and the $b$ series with the largest full core but with peaks outside this core [17]. The non-redundancy constraint of Golay's search is not only no longer required as soon as sub-apertures are phased, but is even a detrimental constraint: although the Golay PAs have maxi-

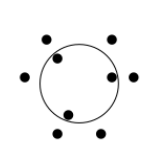

G9a [45]

$3.61(5.00)$

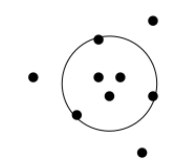

G9b [51]

$4.36(6.08)$
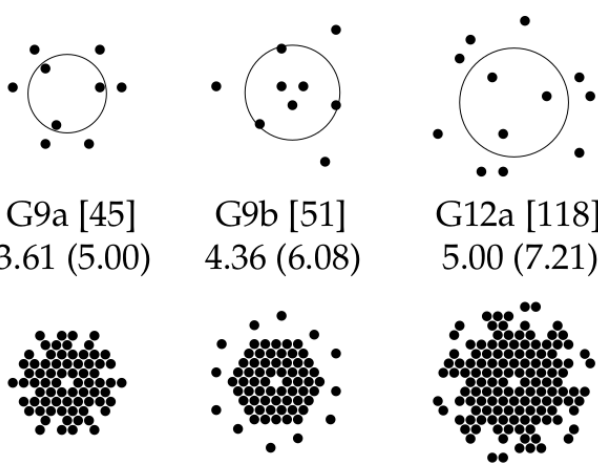

G12a [118]

$5.00(7.21)$

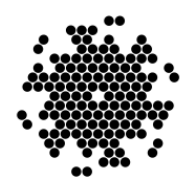

G12b. [135]
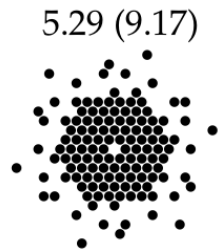

Fig. 2. Some Golay PAs (top) and PAAs (bottom). Text annotations are detailed after $\ddagger$ in the caption of Fig. 3.

mum compactness, they are not strictly compact in the sense of the frequency coverage previously discussed. Indeed, the $a$ series has a reduced PRL because of the frequency holes, and the $b$ series suffers from aliasing noise introduced by baselines significantly longer than required.

This motivated the exhaustive search we performed on a triangular lattice, similarly to Golay, but with a different metric as the quality criterion. The selected approach is, for a given number $N$ of sub-apertures, to compute the PRL for each of the subapertures' positions, and then to identify the configurations with the largest PRL, even if these configurations include some unavoidable redundancy. PAs with $120^{\circ}$ invariance are selected, leading to highly symmetric PAAs with $60^{\circ}$ invariance. PAs with a central point (such as G7a,b [17]) are not considered, to leave room for some combining optics in the aperture center. The first of these maximum compactness PAs (C-PAs) are shown in Fig. 3 for $N \leq 21$, grouped by number of apertures $N$, and ordered by decreasing $D_{\text {PRL }}$ (MTF performance), then by increasing maximum baseline $B_{\mathrm{M}}$ (structure cost), then by increasing moment of inertia $I$ (pointing cost).

Figure 3 shows that compact PAs with a small redundancy exist. For $N=9, \mathrm{C} 9 \mathrm{a}$ is the only PA with the largest core, a 5-sided hexagon with $D_{\mathrm{PRL}}=4.36$. C9a has the same PAA core support as G9b (Fig. 2) but $B_{\mathrm{M}}$ is $33 \%$ smaller. Other PAs have $D_{\text {PRL }} \leq 3.61$, except $C 9 \mathrm{~b}$ with $D_{\mathrm{PRL}}=4$. For $N=12$, four PAs have a 6-sided hexagonal PAA, but some PAAs even have points on the next ring, which give them the highest $D_{\mathrm{PRL}}=5.29$. With $N=15$, eight PAAs are larger than the 7-sided hexagon. For $N=18$, the largest PAA is the 9-sided hexagon. It can be noted that best PAs shapes are often elbows (C12b, C15b, C18b), or trapezes (C12a, C15a, C18a) with a slightly smaller moment of inertia. For $N=21$, best PAs are clustered along a ring. For best $C$ arrays, in Fig. 3:

$D_{\mathrm{PRL}} \simeq 0.43 N P . \quad$ (at least for $\left.N \in\{9,12,15,18,21\}\right)$.

This empirical scaling law shows that C-PAs are 33\% better than regular polygon PAs, for which $D_{\mathrm{PRL}} \simeq N P / \pi$.

The value of $\mathrm{MTF}_{\min }$ can be approximated as a function of $N$ and the dilution ratio $d_{L}$ only. For a C-PA, from the MTF value at the center of any triangle formed by the surrounding non-redundant maxima [white spot in Fig. 1(b)] and the MTF $f_{0}$ of a unit-diameter sub-aperture $\left(f_{0}(0)=1\right)$, we obtain:

$$
\mathrm{MTF}_{\min } \simeq \frac{1}{N} f\left(d_{L}\right), \text { with } f\left(d_{L}\right)=3 f_{0}\left(d_{L} / \sqrt{3}\right) .
$$

Inversion of Eq. (2) is simple: for circular sub-apertures, the $f$ function [18] can be well approximated by a linear fit versus 


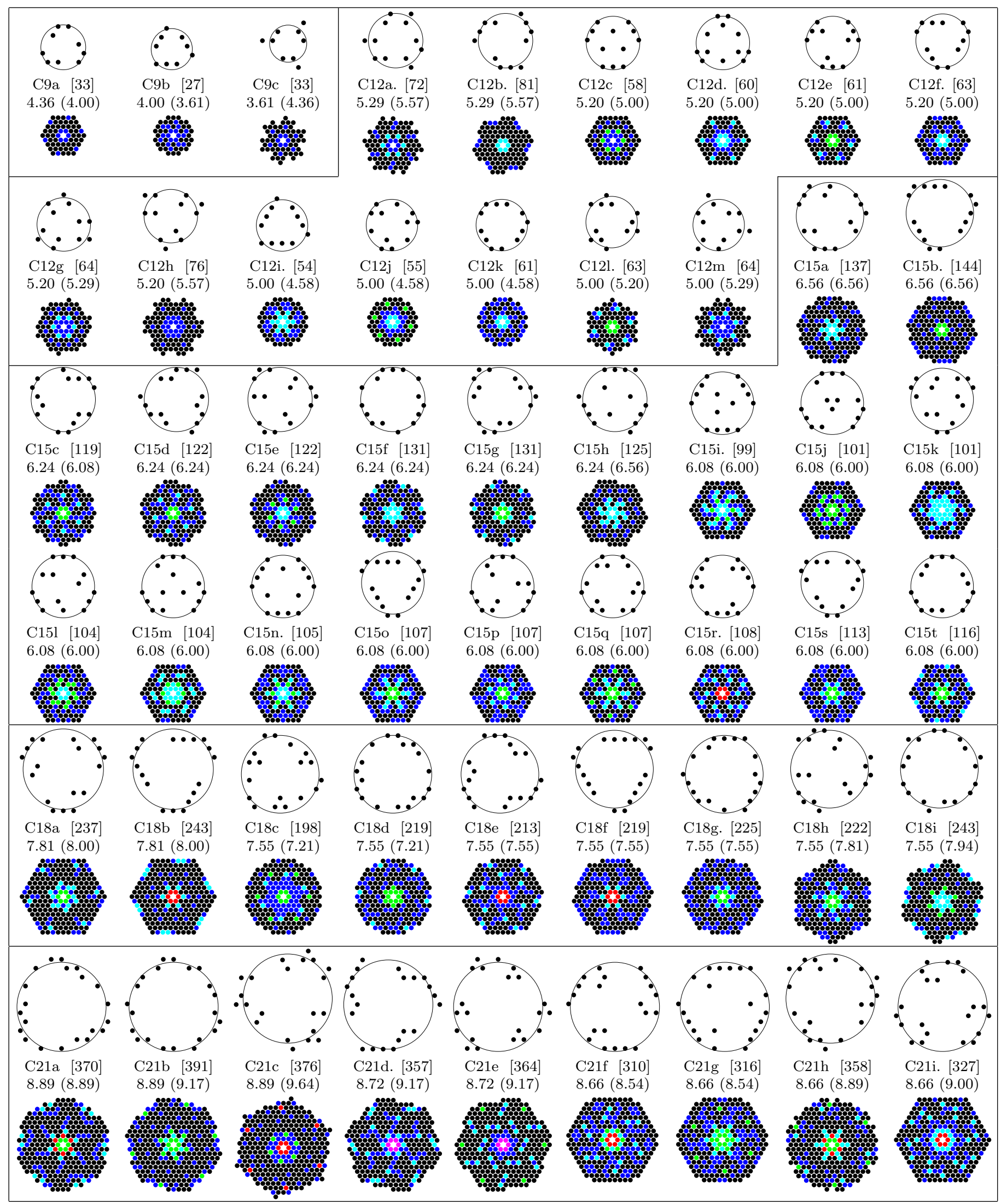

Fig. 3. First maximum compactness PAs with 9, 12, 15, 18 and 21 sub-apertures in successive cells. The redundancy amount is given by the color: $1,2,3,4,5,6$. $\ddagger$ PAs (top, with PRL circle), drawn with $d_{L}=2.2$ to separate fringe peaks in the PAA support (bottom,

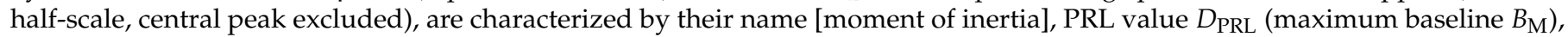
with $P=1$. The few PAs whose center is on the lattice (unlike in Fig. 1) have a dot after their names. 


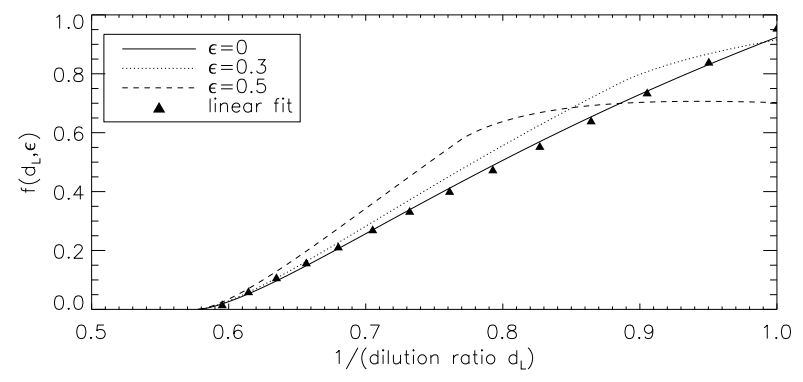

Fig. 4. Evolution of $\mathrm{MTF}_{\min }$ through the $f$ function in Eq. (2) versus the dilution ratio $d_{L}$ and the linear central obscuration $\epsilon$.

$d_{L}^{-1}$ (Fig. 4); $d_{L}^{-1}$ directly derives through Eq. (1) from another high-level parameter, and the fill-factor $d_{S}=N D^{2} / D_{\text {PRL }}^{2}$ :

$$
0.588+0.431 N \mathrm{MTF}_{\min } \simeq d_{L}^{-1} \simeq 0.43 \sqrt{N d_{S}} .
$$

Equations. (1) and (3) allow one to quickly configure an optimal aperture from high-level constraints such as $N$ and $\mathrm{MTF}_{\min }$ or $d_{S}$. For example, a $D_{\mathrm{PRL}}=10 \mathrm{~m}$ telescope with $\mathrm{MTF} \geq 5 \%$ can be based on C9a, with $P \simeq 2.6 \mathrm{~m}$ [Eq. (1)] and $d_{L} \simeq 1.3$ [Eq. (3)], thus $D \simeq 2 \mathrm{~m}$ sub-apertures; with $\mathrm{C} 18 \mathrm{a}, P \simeq 1.3 \mathrm{~m}, d_{L} \simeq 1.03$ and $D \simeq 1.25 \mathrm{~m}$ (Fig. 5). The fill-factor $d_{S}$ is slightly less than $30 \%$ in both cases, but C18a has very close sub-apertures and a lower MTF average. The $\mathrm{C} 15 \mathrm{~b}$ array gives slighty larger apertures than $\mathrm{C} 18 \mathrm{a}(\mathrm{D} \simeq 1.4 \mathrm{~m})$, but a larger spacing $\left(d_{L}=1.1\right)$, nearly linear arms, and a higher MTF average.

In conclusion, we have proposed a simple methodology to define the aperture configuration of a SAIS: system requirements impose the Frequency Domain of Interest over which $\mathrm{MTF}_{\min }$ is to be maximized, imaging requires minimum wavefront errors, and manufacturing pleads for identical sub-apertures. A systematic search for configurations with $120^{\circ}$ invariance on a triangular lattice with maximum PRL led to the ones shown in Fig. 3 for $N=9$ to 21 sub-apertures. From this catalog and other constraints (location along a beam or near a ring), an aperture configuration can be selected, possibly by iterating on $N$. The two free parameters, the grid pitch, and the sub-aperture diameter, then directly derive from two high-level specifications, the sought resolution (PRL) and the MTF level or the fill-factor $d_{S}$, through Eqs. (1) and (3), respectively, which are typically set from the photometric budget. Because compactness is required
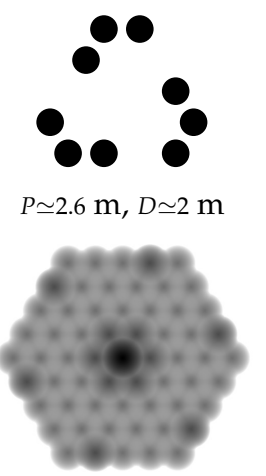

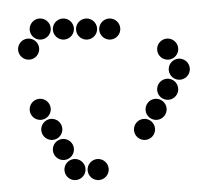

$P \simeq 1.55 \mathrm{~m}, D \simeq 1.4 \mathrm{~m}$

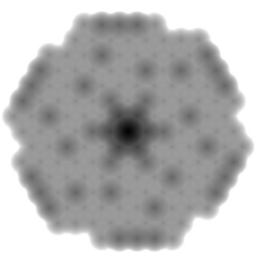

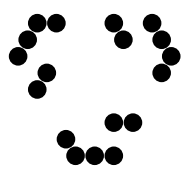

$P \simeq 1.3 \mathrm{~m}, D \simeq 1.25 \mathrm{~m}$

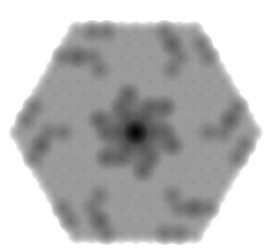

Fig. 5. Apertures C9a, C15b, C18a (top) and associated MTFs (bottom, logarithmic scale) with $D_{\mathrm{PRL}}=10 \mathrm{~m}$ and $\mathrm{MTF}>5 \%$. for a wide-field imager and non-redundancy cannot be simultaneously met, these configurations are slightly redundant, but more efficient for phased arrays than other ones since they provide the smallest baselines, thus less or no aliasing, and nearlyniform MTFs. Eq. (3) shows that the change in MTF level with the fillfactor is more complex than linear, which should slightly modify some quantitative conclusions of [15]. The arrays in Fig. 3 can be used as given or as starting points for a refined optimization based on continuous positions, taking into account the weighting by the observed object spectrum and the exact deconvolution algorithm $[18,24]$. They can also be used with larger dilutions to image compact objects with hypertelescopes [25].

\section{FUNDING}

ONERA (PRF MÉTOPE); Centre National d'Etudes Spatiales (CNES).

\section{REFERENCES}

1. A. H. Fizeau, C. R. Acad. Sci. Paris (1868).

2. J. P. Lloyd, F. Martinache, M. J. Ireland, J. D. Monnier, S. H. Pravdo, S. B. Shaklan, and P. G. Tuthill, Astrophys. J. Lett. 650 (2), L131 (2006).

3. GRAVITY Collab., Astron. Astrophys. 602, A94 (2017).

4. M. Mesrine, E. Thomas, S. Garin, P. Blanc, C. Alis, F. Cassaing, and D. Laubier, Proc. SPIE 10567, p. 105670B (2017).

5. A. B. Meinel and M. P. Meinel, Opt. Eng. 41, 1983 (2002).

6. H. R. Schmitt, D. Mozurkewich, J. T. Armstrong, A. M. Jorgensen, E. K. Baines, S. R. Restaino, R. B. Hindsley, and G. van Belle, Proc. SPIE 8445, 84452P (2012).

7. N. A. Massie, Y. Oster, G. Poe, L. Seppala, and M. Shao, Appl. Opt. 31, 447 (1992).

8. A. B. Meinel and M. P. Meinel, Appl. Opt. 43, 601 (2004).

9. R. L. Kendrick et al., Appl. Opt. 45, 4235 (2006).

10. T. Su, G. Liu, K. E. Badham, S. T. Thurman, R. L. Kendrick, A. Duncan, D. Wuchenich, C. Ogden, G. Chriqui, S. Feng, J. Chun, W. Lai, and S. J. B. Yoo, Opt. Express 26, 12801 (2018).

11. W. T. Rhodes, Appl. Opt. 51, A11 (2012).

12. K. Matthews, A. M. Ghez, A. J. Weinberger, and G. Neugebauer, Pub. Astron. Soc. Pacific 108, 615 (1996).

13. M. Schöller, EAS Publications Series 28, 1 (2008).

14. É. Thiébaut and J. Young, J. Opt. Soc. Am. A 34, 904 (2017).

15. J. R. Fienup, Proc. SPIE4091, 43 (2000).

16. J. E. Harvey and R. A. Rockwell, Opt. Eng. 27, 762 (1988).

17. M. J. E. Golay, J. Opt. Soc. Am. 61, 272 (1971).

18. L. M. Mugnier, G. Rousset, and F. Cassaing, J. Opt. Soc. Am. A 13, 2367 (1996).

19. J. L. Flores, G. Paez, and M. Strojnik, Appl. Opt. 38, 6010 (1999).

20. A. B. Meinel, M. P. Meinel, and N. J. Woolf, in Applied optics and optical engineering (1983), vol. IX.

21. P. S. Salvaggio, J. R. Schott, and D. M. McKeown, Appl. Opt. 55, 3182 (2016).

22. A. Liu, L. Gao, and M. Xiao, Opt. Eng. 56, 073109 (2017).

23. L. Mugnier, F. Cassaing, G. Rousset, F. Baron, V. Michau, I. Mocœur, B. Sorrente, and M.-T. Velluet, in Proc. OPTRO Symposium (2005).

24. L. Mugnier, F. Cassaing, B. Sorrente, F. Baron, M.-T. Velluet, V. Michau, and G. Rousset, in 5th International Conference On Space Optics, (ESA, 2004), SP-554, pp. 181.

25. A. Labeyrie, Astron. Astrophys. Suppl. Ser. 118, 517 (1996). 